\title{
Selective dissociation of the spectator Auger final states in $\mathrm{O}_{2}$ molecules
}

\author{
Lifang Tian, ${ }^{1}$ Bocheng Ding, ${ }^{1}$ Christophe Nicolas, ${ }^{2}$ Ruichang Wu, ${ }^{1}$ Yunfei Feng, ${ }^{1}$ Minna Patanen, ${ }^{2,3}$ Saikat Nandi, ${ }^{2}$ \\ John Bozek, ${ }^{2}$ Catalin Miron $\odot,{ }^{4}$ and Xiao-Jing Liu $\odot^{1, *}$ \\ ${ }^{1}$ School Physical Science and Technology, ShanghaiTech University, Shanghai 201210, China \\ ${ }^{2}$ Synchrotron SOLEIL, l'Orme des Merisiers, Saint-Aubin, BP 48, 91192 Gif-sur-Yvette Cedex, France \\ ${ }^{3}$ Nano and Molecular Systems Research Unit, Molecular Materials Research Community, Faculty of Science, University of Oulu, \\ P.O. Box 3000, 90014 Oulu, Finland \\ ${ }^{4}$ Université Paris-Saclay, CEA, CNRS, LIDYL, 91191 Gif-sur-Yvette, France
}

(Received 10 January 2021; revised 21 March 2021; accepted 22 March 2021; published 8 April 2021)

\begin{abstract}
Molecular cationic states with two valence holes and one $n$-Rydberg electron can be created after spectator Auger decay. Unraveling these states' dissociation is often very challenging due to the frequent occurrence of conical intersections between cationic potential energy curves. Here, based on an advanced analysis of the experimental multicoincidence data obtained after $\mathrm{O} 1 s$ core excitation in $\mathrm{O}_{2}$, we achieved an energy-resolution better than we recently exploited in Phys. Rev. A 99, 022511 (2019). We therefore revealed a group of weak channels in the two-dimensional energy-correlation map between the coincident resonant Auger electron and ion in addition to the previously reported strong ones. The fragments in the identified weak channels contain only outmost electrons in valence orbitals; in contrast, the fragments in the strong channels contain an outmost electron in a $n^{\prime}$-Rydberg orbital. Compared with the strong channels, the weak channels preferentially occur at smaller principal quantum number $n$. It indicates that the electron orbital size tends to be conserved during the dissociation process. These weak features are suggested to be created by the Rydberg-valence mixing between the molecular spectator Auger final state and the very dissociative molecular cationic states without the Rydberg electron. A tendency to orbital selection is also suggested in the Rydberg-valence mixing.
\end{abstract}

DOI: 10.1103/PhysRevA.103.043103

\section{INTRODUCTION}

Molecular core excitation often causes molecular deformation [1], and several situations may occur [2,3]. In the first case, when an electron is excited from a core orbital to a strongly antibonding valence one, the repelling force in the neutral molecule is so strong that light nuclei can move significantly far from the equilibrium ground state geometry during the core-hole lifetime. In extreme cases, atomic lines are observed in the resonant Auger electron spectra [4-6], implying that the electronic decay takes place in an excited atom fully separated from the rest of the molecule. In another possible case, as discussed here in the context of the $\mathrm{O}_{2}$ molecule, when an electron is excited to a Rydberg orbital, the molecular structure does not change dramatically because both core and Rydberg orbitals are chemically weakly bonding $[7,8]$. Spectator Auger decay dominates the electronic decay processes and leaves the molecule to a two-hole-one-electron (2h1e) cationic state with two valence holes and one electron in a Rydberg orbital [9-12]. Such 2h1e states are often dissociative because the Rydberg electron provides only partial screening for the repulsive force in the dicationic core. The dissociation dynamics depends on the orbitals involved, which makes the $2 \mathrm{~h} 1 \mathrm{e}$ states interesting systems to understand molecular bond breaking.

\footnotetext{
*liuxj@shanghaitech.edu.cn
}

Molecular dissociation after excitation or ionization of one or two electrons was well investigated in the last decades. In a superexcited $\mathrm{O}_{2}$ molecule, where an inner valence electron is excited so that the energies of the excited states lie above the first ionization threshold, there are always neutral dissociation pathways leading to bond breaking in the ionic core, with the Rydberg electron acting as a spectator [13-15]. After the dissociation, the effective principal quantum number $n^{*}$ of the molecular Rydberg state can be kept in the atomic fragment, as demonstrated by the observation of atomic lines in the fluorescence spectrum $[14,16]$.

The ionization of an inner valence orbital may induce molecular dissociation or predissociation and end up with a neutral atom and a singly charged ion [17-19]. The potential energy curves of singly ionized states can be relatively easily calculated [20,21]. Dicationic states can be created by electron impact, photoionization, or Auger decay [22-25], and their potential energy curves were also calculated $[20,26,27]$ to follow the dissociation and predissociation along them.

Compared with the abovementioned cationic and excited states, the dissociation of $2 \mathrm{~h} 1 \mathrm{e}$ states were much less investigated. The reason is twofold: (i) from the experimental point of view, either it is challenging to achieve high energy resolution for the high kinetic resonant Auger electron in the coincident experiment [28-31], or it is hard for the highresolution resonant Auger electron spectroscopy to directly probe the potential energy curve outside the Franck-Condon region [11]. (ii) From the theoretical point of view, if one 
wants to go beyond the approximation that the $2 \mathrm{~h} 1 \mathrm{e}$ states are described by simply attaching one Rydberg electron to the dicationic core, the number of potential energy curves will increase by more than 10 times. Moreover, during the dissociation where the $2 \mathrm{~h} 1 \mathrm{e}$ state evolves into a singly charged ion and a neutral atom with a Rydberg electron, the charge of the ionic core felt by the excited electron changes from 2 to 1 . Thus, this simple approximation will fail as the internuclear distance increases at a certain value. Furthermore, there are a lot of conical intersections among the potential energy curves of the $2 \mathrm{~h} 1 \mathrm{e}$ states due to spin-orbit coupling or configuration interaction. To the best of our knowledge, the detailed ab initio calculated potential energy curves of $\mathrm{O}_{2}$ 2h1e states have not been reported in the literature.

In our very recent paper [32], we described the strongest channels in the $\mathrm{O}_{2}$ dissociation into $\mathrm{O}^{+}$and $\mathrm{O}$ with a Rydberg electron following $\mathrm{O} 1 s \sigma \rightarrow\left(1 s^{-14} \Sigma_{u}^{-}\right) 4 p \sigma /$ $\left(1 s^{-12} \Sigma_{u}^{-}\right) 3 p^{\prime} \sigma$ excitation. Many more dissociation channels are opened compared with the dicationic $\mathrm{O}_{2}^{++}$states [22]. On the top right of Fig. 3(a) in Ref. [32], there are some very weak features that were left out of the discussion. However, after a very careful additional analysis, we found out that they carry signatures of additional dissociation channels. In this paper, we reanalyzed the experimental data to improve the energy resolution. It turns out that these weak features correspond to dissociation into $\mathrm{O}^{+} / \mathrm{O}$ without a Rydberg electron. The Rydberg can shrink or collapse into a valence orbital during the dissociation process. This observation points toward selectivity on the orbital size in the dissociation processes.

\section{EXPERIMENTAL METHOD}

Since the experimental method has already been described in Ref. [32], only a very brief description is given here. The experiments were carried out using the EPICEA setup at the PLEIADES beamline [33] at Synchrotron SOLEIL in SaintAubin, France. This setup was composed of a double-toroidal electron analyzer (DTA) [34-36] and an ion time of flight (TOF) spectrometer.

The DTA selected the electrons within the polar angle of $54 \pm 3^{\circ}$ and the azimuthal angles ranging from $0^{\circ}$ to $360^{\circ}$ with respect to the polarization vector of the photon beam. Only electrons with the kinetic energy comprised in a window of about $12 \%$ of the pass energy of the DTA reached the detector and got recorded. The photon energy was set at $541.8 \mathrm{eV}$, and the light polarization was parallel to the symmetry axis of the setup. The DTA was operated around the central kinetic energy of $503 \mathrm{eV}$ with a pass energy of $80 \mathrm{eV}$, resulting in an electron energy resolution of $0.7 \mathrm{eV}$.

Mounted opposite to the DTA, there was an ion TOF spectrometer with a position-sensitive detector allowing one to measure the ion momentum. A pulsed $\pm 100 \mathrm{~V}$ was triggered by the detection of an Auger electron and pushed the relevant ions toward the ion detector. The mass resolution for nonenergetic ions was $\sim 1000$, the ion momentum resolution was $\sim 1.4$ a.u, and the ion energy resolution was $\sim 0.3 \mathrm{eV}$. A random signal generator was used to simulate the false coincident events, and their contribution was subtracted from the data according to the method described by Prümper et al. [37].

Like the reaction microscope [38,39], a large volume of multicoincidence data were recorded, and various kinds of spectra were created related to the spectrometer hardware and the physics. Following our recent paper [32], we found that the rings in the acquired electron image could be slightly centershifted from each other and slightly jagged, which might be due to a tiny misalignment and surface nonuniformity of the electrodes in the DTA. Using two such rings as references and assuming that the effect was linearly proportional to the radius on the image, all the rings were corrected to be as smooth and concentric as possible. As a result, the electron energy resolution was improved by $\sim 8 \%$ compared with this prior paper [32].

\section{RESULTS AND DISCUSSION}

We plot the ion kinetic energy release (KER; sum of the kinetic energies of the $\mathrm{O}$ and $\mathrm{O}^{+}$fragments) against the Auger electron kinetic energy in Fig. 1(a). As a result of the improved energy resolution after time-consuming fine-tuning of parameters in the data analysis, the highest number of count is 1035 here as compared with 897 in fig. 3(a) in Ref. [32]. Two sets of features are seen in Fig. 1(a): the features in the lower left part are strong, while the ones in the upper right are very weak. By zooming in the color scale 13 times and redisplaying the data in Fig. 1(b), the weak features are much more visible and numbered from 1 to 9 . All the features are grouped on diagonal lines with a slope of -1 caused by the energy conservation law:

$$
\begin{aligned}
E(h v)= & E_{k}\left(e_{\text {Auger }}^{-}\right)+E_{\mathrm{KER}}+E_{i}(\mathrm{O}) \\
& +E_{i}\left(\mathrm{O}^{+}\right)+E_{\mathrm{IP}}(O)+E_{D}\left(\mathrm{O}_{2}\right)
\end{aligned}
$$

Here, $E(h v)$ is the photon energy, $E_{k}\left(e_{\text {Auger }}^{-}\right)$is the Auger electron kinetic energy, and $E_{\mathrm{KER}}$ is the KER, $E_{i}(\mathrm{O})$ and $E_{i}\left(\mathrm{O}^{+}\right)$ are the excitation energies of the atom and ion, respectively, $E_{\mathrm{IP}}(\mathrm{O})$ is the oxygen-atom ionization potential, and $E_{D}\left(\mathrm{O}_{2}\right)$ is the dissociation energy of the $\mathrm{O}_{2}$ molecule in the ground state. The dissociation limit energy $E_{\text {diss }}$ is defined as

$$
\begin{aligned}
E_{\mathrm{diss}} & =E(h v)-E_{k}\left(e_{\text {Auger }}^{-}\right)-E_{\mathrm{KER}} \\
& =E_{D}\left(\mathrm{O}_{2}\right)+E_{\mathrm{IP}}(\mathrm{O})+E_{i}(\mathrm{O})+E_{i}\left(\mathrm{O}^{+}\right) .
\end{aligned}
$$

In Fig. 1, the higher the lines intercept the axis, the lower their $E_{\text {diss. }}$.

The observed dissociation limit energies $E_{\text {diss }}$ of various $\mathrm{O}^{+}+\mathrm{O}$ channels are calculated using Eq. (2), and the assignments are achieved using Eq. (3) with the energy values from the National Institute of Standards and Technology atomic database [40]. The various $E_{\text {diss }}$ are represented by the red dashed diagonal lines in Figs. 1(a) and 1(b). The assignment of $E_{\text {diss }}$ in Fig. 1(a) was already listed in Table I in Ref. [32], where the $\mathrm{O}$ atomic fragment contains a Rydberg electron. Here, we label them as R1, R2, R3, and R4. On the other hand, the assignments of $E_{\text {diss }}$ in Fig. 1(b) are listed in Table I, where both $\mathrm{O}^{+}$and $\mathrm{O}$ contain only valence electrons and no Rydberg electron. They are labeled as V1, V2, and V3. In Table I, we also display the other dissociation limits for which both $\mathrm{O}^{+}$ 


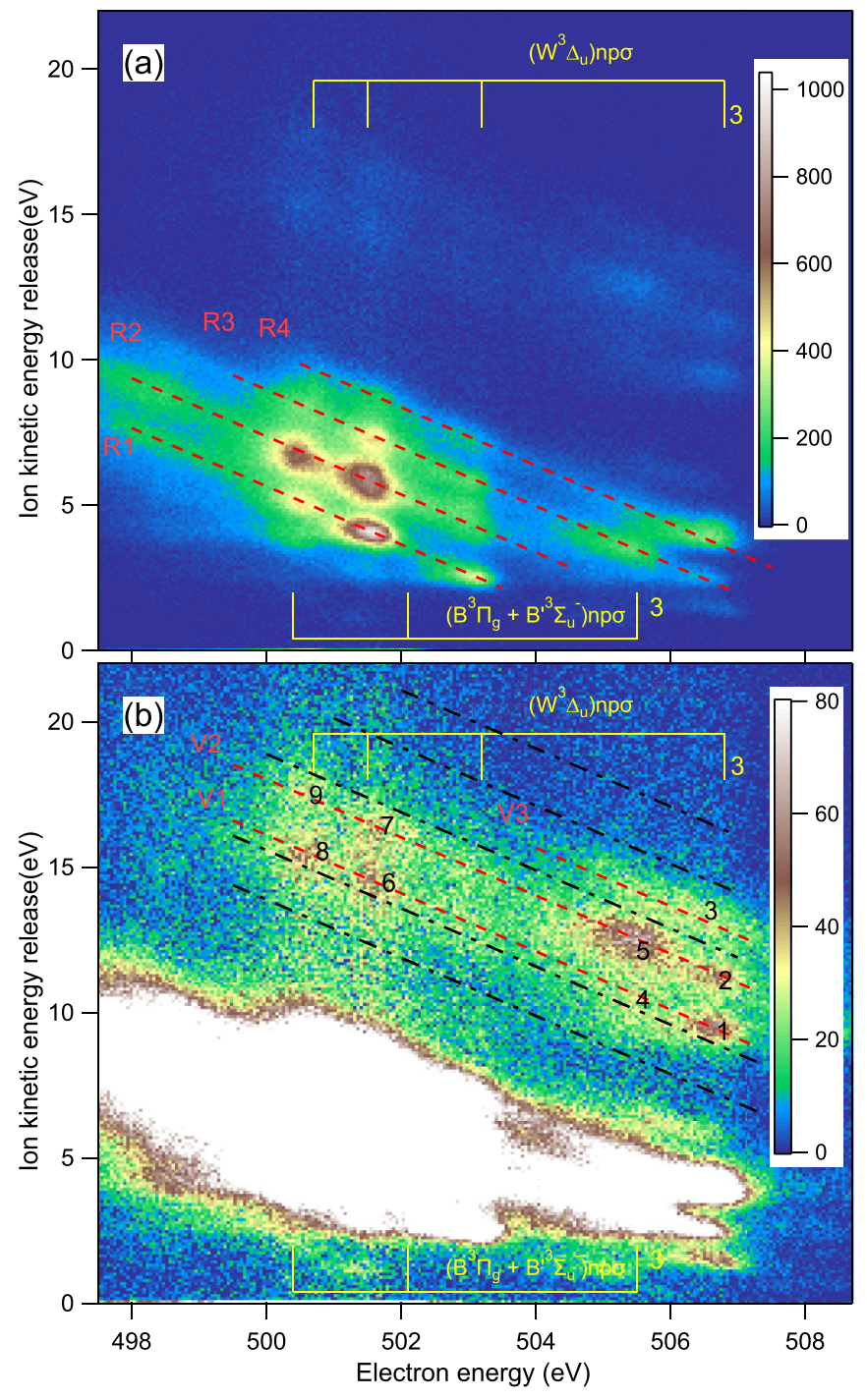

FIG. 1. Correlation between the kinetic energy of the spectator Auger electron and the kinetic energy release of the fragments $\mathrm{O}^{+} / \mathrm{O}$. To highlight the weak features, the color scale in (a) is zoomed in 13 times and redisplayed in (b). The red diagonal dashed lines represent groups of dissociation limits after spectator Auger decay. They are labeled as R1-R4 in (a), as the $\mathrm{O}$ atom contains a Rydberg electron, and as $\mathrm{V} 1-\mathrm{V} 3$ in (b), as the $\mathrm{O}$ atom has only valence electrons. The black diagonal dashed-dotted lines indicate the missing dissociate limits. The assignments of the spectator Auger lines were adopted from Tanaka et al. [11].

and $\mathrm{O}$ only contain valence electrons, as indicated by circles in the table and by five black dashed-dotted lines in Fig. 1(b); however, they do not show observable intensities.

It is obvious that the features in the lower left part of the coincidence map, where the Rydberg electron remains in the atomic fragment, are of much higher intensity than those in the upper right region, as seen in Fig. 1(a). This intensity difference can be qualitatively explained by examining the steps of excitation, Auger decay, and dissociation. First, the photon energy was set at $541.8 \mathrm{eV}$; although both $\mathrm{O} 1 s^{-1}\left({ }^{4} \Sigma_{u}^{-}\right) 4 p \sigma$ and $\mathrm{O} 1 s^{-} 1\left({ }^{2} \Sigma_{u}^{-}\right) 3 p^{\prime} \sigma$ contribute to the population of the excitation, $\mathrm{O} 1 s^{-1}\left({ }^{4} \Sigma_{u}^{-}\right) 4 p \sigma$ dominates. Second, the shakedown
TABLE I. The assignment of the dissociation limits $E_{\text {diss }}$ for the features observed in Fig. 1(b) for which the fragments $\mathrm{O}^{+}+\mathrm{O}$ contain only valence electrons. $\bigcirc$ symbols are used to mark missing features in Fig. 1(b). The energy unit is electronvolts.

\begin{tabular}{|c|c|c|c|c|c|c|}
\hline \multirow[b]{2}{*}{$\mathrm{O}$} & \multicolumn{2}{|c|}{$\left(\mathrm{O}^{+}\right){ }^{4} S$} & \multicolumn{2}{|c|}{$\left(\mathrm{O}^{+}\right)^{2} D$} & \multicolumn{2}{|c|}{$\left(\mathrm{O}^{+}\right)^{2} P$} \\
\hline & $E_{\text {diss }}$ & Label & $E_{\text {diss }}$ & Label & $E_{\text {diss }}$ & Label \\
\hline${ }^{3} P$ & 18.72 & 0 & 22.04 & $3 ; \mathrm{V} 3$ & 23.74 & $2,5,7,9 ; \mathrm{V} 2$ \\
\hline${ }^{1} D$ & 20.69 & 0 & 24.01 & $2,5,7,9 ; \mathrm{V} 2$ & 25.71 & $1,4,6,8 ; \mathrm{V} 1$ \\
\hline${ }^{1} S$ & 22.91 & 0 & 26.23 & $\bigcirc$ & 27.93 & $\bigcirc$ \\
\hline
\end{tabular}

processes are expected to be negligible in the spectator Auger decay according to the relaxation model [11], so the Rydberg electron should have a principal quantum number $n>3$ after the spectator Auger decay. Last, a Rydberg electron would tend to act as a spectator during the dissociation of the molecular ionic core, so it is expected that one of the fragments will carry a Rydberg electron after the dissociation.

However, there are two puzzles in Fig. 1: the appearance of the dissociation limit $\mathrm{V} 1\left[\mathrm{O}\left({ }^{1} D\right)+\mathrm{O}^{+}\left({ }^{2} P\right)\right]$ and the gap between the groups " $R$ " and the group "V." As Lundqvist et al. [22] reported, three dissociation pathways for $\mathrm{O}_{2}{ }^{2+}: \mathrm{O}_{2}{ }^{2+}\left(\mathrm{W}^{3} \Delta_{u}\right) \rightarrow \mathrm{O}^{+}\left({ }^{4} S\right)+\mathrm{O}^{+}\left({ }^{2} D\right)$, $\mathrm{O}_{2}{ }^{2+}\left(\mathrm{B}^{\prime}{ }^{3} \Sigma_{u}^{-}\right) \rightarrow \mathrm{O}^{+}\left({ }^{4} S\right)+\mathrm{O}^{+}\left({ }^{2} D\right)$, and $\mathrm{O}_{2}{ }^{2+}\left(\mathrm{B}^{3} \Pi_{g}\right) \rightarrow$ $\mathrm{O}^{+}\left({ }^{4} S\right)+\mathrm{O}^{+}\left({ }^{4} S\right), \mathrm{O}^{+}\left({ }^{2} P\right)$ cannot be created if we assume that the Rydberg electron acts purely as a spectator of the dissociation of the dicationic core $\mathrm{O}_{2}{ }^{2+}$. The absence of the dissociation limit $\mathrm{O}\left({ }^{1} S\right)+\mathrm{O}^{+}\left({ }^{2} D\right)$ in the gap is also puzzling because the interaction between the Rydberg electron and the ionic core always tends to open new dissociation channels [32]. These two puzzles can be explained by the Rydbergvalence mixing. Because the energy difference between the $3 \sigma_{u}$ antibonding orbital and the $3 p \sigma$ Rydberg orbital is small in an $\mathrm{O}_{2}$ molecule, as shown by Yagishita et al. [7], the $3 p \sigma$ orbital can transfer to the strongly antibonding $3 \sigma_{u}$ orbital. After Rydberg-valence mixing, the molecular potential curves are more repulsive, and the kinetic energies of fragments are higher, as indicated by Fig. 1. Since both potential curves before and after mixing are dissociative, the channel mixing would most probably happen between two states with the same symmetry in the Franck-Condon region, where the kinetic energy is low. Beebe et al. [20] calculated a lot of potential curves of $\mathrm{O}_{2}{ }^{+}$. Among them, we chose very repulsive potential curves which have correct binding energy in the Franck-Condon region and matching symmetry with the dissociation limits given in Table I, so the ${ }^{2,4} \Delta_{g}$, the ${ }^{2,4} \Pi_{u}$, and the ${ }^{2,4} \Sigma_{g}^{-}$from Beebe et al. [20] are the most probable candidates. As the result, only the third, fourth, fifth, sixth, and seventh limits should exist, which agrees reasonably well with the observed third, fifth, sixth, and seventh limits in Table I. More importantly, it can be seen from Fig. 1 that, while the features with the principal quantum number $n=(5,6)$ dominate the dissociation limits $\mathrm{R} 1-\mathrm{R} 4$, the features with $n=3$ are significantly enhanced for the dissociation limits V1-V3. This is because the Rydberg-valence mixing favors a smaller principal quantum number $n$.

To highlight the details, we plotted the partial spectator Auger electron spectra corresponding to the 

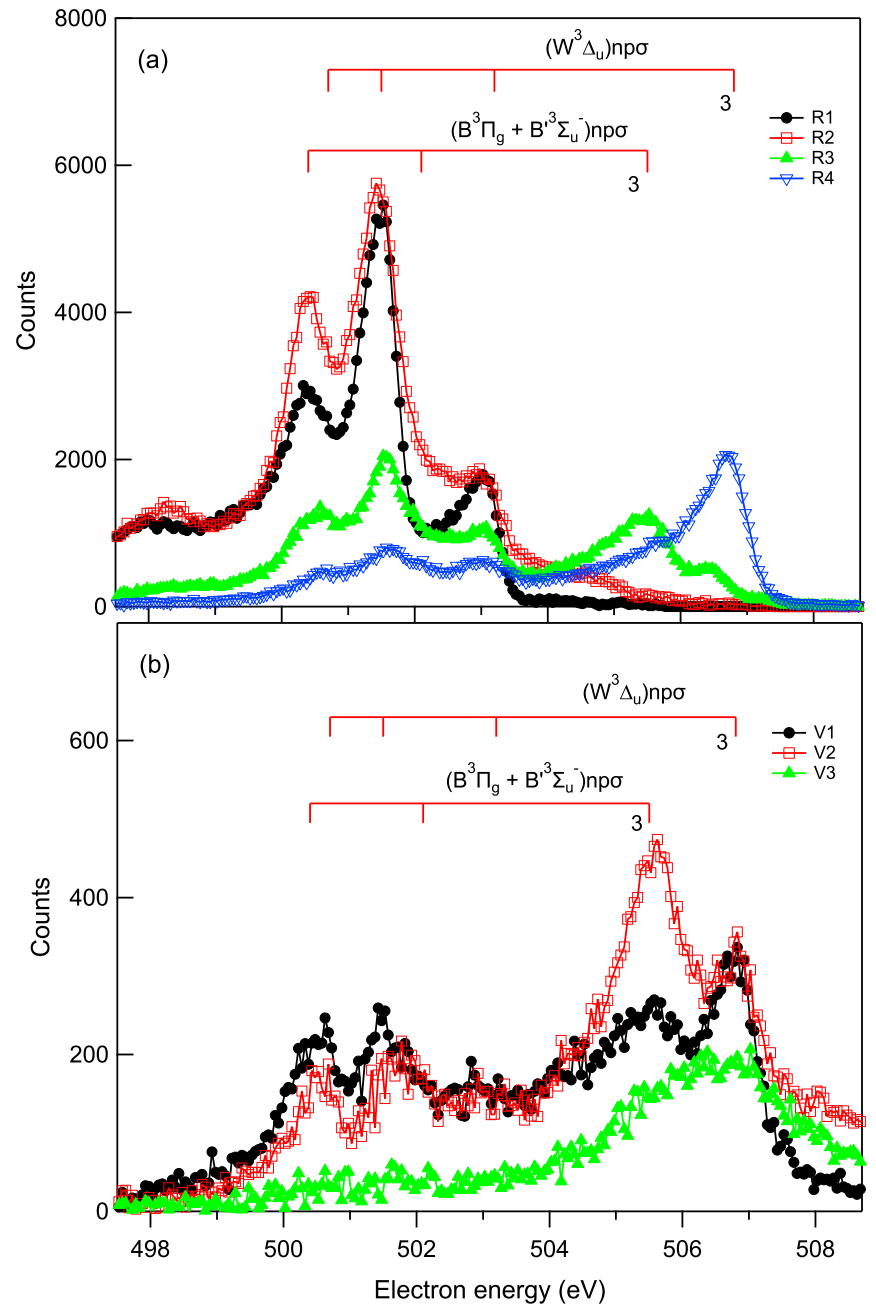

FIG. 2. The partial resonant Auger electron spectra by filtering the data by selecting different dissociation limits. The electron spectra related to R1-R4 are represented by black filled circles, red hollow squares, green filled triangles, and blue hollow triangles in (a). The electron spectra related to V1-V3 are represented by black filled circles, red hollow squares, and green filled triangles in (b), respectively.

dissociation limits R1-R4 and V1-V3 in Figs. 2(a) and 2(b), respectively. It can be noted that the smaller principal quantum number $n$ is enhanced even when the dissociation limit changes from $\mathrm{R} 1 \rightarrow \mathrm{R} 4$ or $\mathrm{V} 1 \rightarrow \mathrm{V} 3$.
The observed propensity in R1-R4 can be explained as follows. Since the Rydberg states have the effective quantum number $n^{*}>2.6$ [11], the typical radius of the Rydberg orbital is larger than $1.8 \AA$. This radius lies beyond the crossing point of the potential energy curves of $\mathrm{O}_{2}{ }^{2+}$ [22], which suggests that the dissociation would mainly lead to products with a Rydberg electron attached to one member of the ion pairs $\mathrm{O}^{+}\left({ }^{4} S\right)+\mathrm{O}^{+}\left({ }^{2} D\right)$ or $\mathrm{O}^{+}\left({ }^{4} S\right)+\mathrm{O}^{+}\left({ }^{4} S\right)$. Thus, we can select from the assignments given in table I of Ref. [32] that follow this rule, i.e., $\left[\mathrm{O}^{+}\left({ }^{4} S\right)+\mathrm{O}^{+}\left({ }^{2} D\right)\right](8 s, 7 d, 9 s, 8 d)$ contribute to $\mathrm{R} 1,\left[\mathrm{O}^{+}\left({ }^{4} S\right)+\mathrm{O}^{+}\left({ }^{2} D\right)\right](4 s, 3 d, 4 p, 5 s, 4 d, 6 s, 5 d)$ contribute to $\mathrm{R} 2,\left[\mathrm{O}^{+}\left({ }^{4} S\right)+\mathrm{O}^{+}\left({ }^{2} D\right)\right] 3 p$ and $\left[\mathrm{O}^{+}\left({ }^{4} S\right)+\right.$ $\left.\mathrm{O}^{+}\left({ }^{4} S\right)\right](7 s, 6 d)$ contribute to $\mathrm{R} 3$, and $\left[\mathrm{O}^{+}\left({ }^{4} S\right)+\mathrm{O}^{+}\left({ }^{2} D\right)\right] 3 s$ contribute to R4. The principal quantum number $n$ of the Rydberg electron in the fragment gets smaller by switching the dissociation limits from $\mathrm{R} 1$ to $\mathrm{R} 4$. The fragment with a smaller principal quantum $n^{\prime}$ of the Rydberg electron would select the molecular state also with smaller principal quantum number $n$ before dissociation.

In the case of V1-V3, according to Hund's rules in atomic states, i.e., the Coulombic repulsion force between electrons gets weaker when the spin quantum number and the orbital angular momentum quantum number of the electronic state increases. This suggests that the size of the outermost orbital gets smaller from $\mathrm{V} 1 \rightarrow \mathrm{V} 3$, thus preferentially selecting the molecular state with a smaller principal quantum number $n$ when the Rydberg-valence mixing happens.

To give a more quantitative result, we obtained the relative branching ratios between all features in Fig. 1 by least-square fitting $[41,42]$ of the curves R1-R4 and V1-V3 in Fig. 2. In the fitting, we assumed that the peaks with different principal quantum number $n$ in a molecular Rydberg series share a common asymmetric Voigt line profile, irrespective of different dissociation limits. All seven curves are fitted simultaneously to get the most reasonable results. After the intensity of the strongest peak corresponding to $\left(\mathrm{W}^{3} \Delta_{u}\right) 5 p \sigma \rightarrow \mathrm{R} 1$ is normalized to 100, all the relative intensities together with the corresponding uncertainties are in Table II.

\section{SUMMARY}

We investigated the dissociation processes of the molecular spectator Auger final states after $\mathrm{O} 1 s \sigma \rightarrow$ $\left(1 s^{-14} \Sigma_{u}^{-}\right) 4 p \sigma /\left(1 s^{-12} \Sigma_{u}^{-}\right) 3 p^{\prime} \sigma \quad$ excitation in an $\mathrm{O}_{2}$ molecule. With an improved resolution, we can highlight the propensity induced by the electron orbitals of the

TABLE II. Relative intensities of all features extracted by least-square fitting of the one-dimensional spectra in Fig. 2 . The values in the square brackets are the uncertainty.

\begin{tabular}{|c|c|c|c|c|c|c|c|}
\hline & $\mathrm{R} 1$ & $\mathrm{R} 2$ & $\mathrm{R} 3$ & $\mathrm{R} 4$ & V1 & $\mathrm{V} 2$ & V3 \\
\hline$\left(\mathrm{W}^{3} \Delta_{u}\right) 3 p \sigma$ & $0[0.6]$ & $0.2[0.2]$ & $6.8[0.4]$ & $40.8[0.6]$ & $8.5[0.6]$ & $12.9[1.0]$ & $9.2[0.8]$ \\
\hline$\left(\mathrm{B}^{\prime}{ }^{3} \Sigma_{u}^{-}+\mathrm{B}^{3} \Pi_{g}\right) 3 p \sigma$ & $0[0.2]$ & $18.5[0.6]$ & $50.4[0.6]$ & $35.0[0.8]$ & $14.4[0.6]$ & $19.6[0.6]$ & $6.9[0.4]$ \\
\hline$\left(\mathrm{W}^{3} \Delta_{u}\right) 4 p \sigma$ & $32.6[0.6]$ & $40.4[0.6]$ & $18.1[0.4]$ & $10.0[0.4]$ & $2.4[0.2]$ & $1.0[0.2]$ & $0.4[0.2]$ \\
\hline$\left(\mathrm{B}^{\prime}{ }^{3} \Sigma_{u}^{-}+\mathrm{B}^{3} \Pi_{g}\right) 4 p \sigma$ & $1.0[0.8]$ & $30.8[0.8]$ & $16.4[0.4]$ & $8.0[0.4]$ & $2.1[0.2]$ & $2.1[0.2]$ & $0.1[0.1]$ \\
\hline $\begin{array}{l}\left(\mathrm{W}^{3} \Delta_{u}\right) 5 p \sigma \\
\left(\mathrm{W}^{3} \Delta_{u}\right) 6 p \sigma+\end{array}$ & $100[1.4]$ & $98.2[1.0]$ & $29.2[0.6]$ & $13.0[0.4]$ & $3.8[0.4]$ & $2.5[0.4]$ & $0.4[0.2]$ \\
\hline$\left(\mathrm{B}^{\prime}{ }^{3} \Sigma_{u}^{-}+\mathrm{B}^{3} \Pi_{g}\right) 5 p \sigma$ & $75.8[3.8]$ & $62.6[3.4]$ & $16.7[1.5]$ & $3.5[0.8]$ & $3.3[1.0]$ & $2.1[0.8]$ & $0.5[0.4]$ \\
\hline
\end{tabular}


parent molecular cation and the fragments, and at the same time the Rydberg-valence mixing can take place between the molecular spectator Auger final state and the highly dissociative molecular cationic states without the Rydberg electron. Due to experimental difficulties to achieve high-resolution Auger electron-ion coincident experiments, the experimental report on such a simple system as $\mathrm{O}_{2}$ are still rare. As a result, the theoretical calculations available are also limited. We hope that our findings will trigger further experimental and theoretical research on these simple and interesting systems.

\section{ACKNOWLEDGMENTS}

X.-J.L. gratefully acknowledges financial support by the National Natural Science Foundation of China (Grant No. 11574020) and Project of Thousand Youth Talents in China. The experiment was performed at the PLEIADES beamline at the SOLEIL Synchrotron, France (Proposal No. 20140796). We are grateful to E. Robert for technical assistance and to the SOLEIL staff for stable operation of the equipment and the storage ring during the experiments. Prof. Kiyoshi Ueda is greatly acknowledged for helpful discussions.
[1] P. Morin, M. Simon, C. Miron, N. Leclercq, and D. L. Hansen, J. Electron Spectrosc. Relat. Phenom. 93, 49 (1998).

[2] C. Miron and P. Morin, in Handbook of High-Resolution Spectroscopy, edited by M. Quack and F. Merkt (John Wiley and Sons, Inc., 2011), pp. 1655-1689.

[3] R. Feifel, Y. Velkov, V. Carravetta, C. Angeli, R. Cimiraglia, P. Salek, F. Gel'mukhanov, S. L. Sorensen, M. N. Piancastelli, A. De Fanis, K. Okada, M. Kitajima, T. Tanaka, H. Tanaka, and K. Ueda, J. Chem. Phys. 128, 064304 (2008).

[4] P. Morin and I. Nenner, Phys. Rev. Lett. 56, 1913 (1986).

[5] O. Björneholm, M. Bässler, A. Ausmees, I. Hjelte, R. Feifel, H. Wang, C. Miron, M. N. Piancastelli, S. Svensson, S. L. Sorensen, F. Gelmukhanov, and H. Ågren, Phys. Rev. Lett. 84, 2826 (2000).

[6] K. Ueda, M. Kitajima, A. De Fanis, T. Furuta, H. Shindo, H. Tanaka, K. Okada, R. Feifel, S. L. Sorensen, H. Yoshida, and Y. Senba, Phys. Rev. Lett. 90, 233006 (2003).

[7] A. Yagishita, E. Shigemasa, and N. Kosugi, Phys. Rev. Lett. 72, 3961 (1994).

[8] J.-i. Adachi, N. Kosugi, and A. Yagishita, J. Phys. B 38, R127 (2005).

[9] M. Neeb, J.-E. Rubensson, M. Biermann, and W. Eberhardt, Phys. Rev. Lett. 71, 3091 (1993).

[10] A. Kivimäki, B. Kempgens, M. N. Piancastelli, M. Neeb, K. Maier, A. Rüdel, U. Hergenhahn, and A. Bradshaw, J. Electron Spectrosc. Relat. Phenom. 93, 81 (1998).

[11] T. Tanaka, R. Feifel, M. Kitajima, H. Tanaka, S. L. Sorensen, R. Sankari, A. De Fanis, M. N. Piancastelli, L. Karlsson, and K. Ueda, Phys. Rev. A 78, 022516 (2008).

[12] T. Gejo, M. Oura, T. Tokushima, Y. Horikawa, H. Arai, S. Shin, V. Kimberg, and N. Kosugi, J. Chem. Phys. 147, 044310 (2017).

[13] M. Ukai, S. Machida, K. Kameta, M. Kitajima, N. Kouchi, Y. Hatano, and K. Ito, Phys. Rev. Lett. 74, 239 (1995).

[14] Y. Hatano, Phys. Rep. 313, 110 (1999).

[15] A. Ehresmann, L. Werner, S. Klumpp, H. Schmoranzer, P. V. Demekhin, B. M. Lagutin, V. L. Sukhorukov, S. Mickat, S. Kammer, B. Zimmermann, and K.-H. Schartner, J. Phys. B 37, 4405 (2004).

[16] A. Ehresmann, P. V. Demekhin, W. Kielich, I. Haar, M. A. Schlüter, V. L. Sukhorukov, and H. Schmoranzer, J. Phys. B 42, 165103 (2009).

[17] A. Lafosse, J. Brenot, A. Golovin, P. Guyon, K. Hoejrup, J. Houver, M. Lebech, and D. Dowek, J. Chem. Phys. 114, 6605 (2001).

[18] Y. Hikosaka, T. Aoto, R. I. Hall, K. Ito, R. Hirayama, N. Yamamoto, and E. Miyoshi, J. Chem. Phys. 119, 7693 (2003).
[19] X. Tang, G. A. Garcia, and L. Nahon, J. Chem. Phys. 148, 124309 (2018).

[20] N. H. F. Beebe, E. W. Thulstrup, and A. Andersen, J. Chem. Phys. 64, 2080 (1976).

[21] K. Tanaka and M. Yoshimine, J. Chem. Phys. 70, 1626 (1979).

[22] M. Lundqvist, D. Edvardsson, P. Baltzer, M. Larsson, and B. Wannberg, J. Phys. B 29, 499 (1996).

[23] J. H. Eland, Chem. Phys. 294, 171 (2003).

[24] D. Mathur, Phys. Rep. 391, 1 (2004).

[25] M. Larsson, P. Baltzer, S. Svensson, B. Wannberg, N. Martensson, A. N. de Brito, N. Correia, M. Keane, M. CarlssonGothe, and L. Karlsson, J. Phys. B 23, 1175 (1990).

[26] D. Edvardsson, S. Lunell, F. Rakowitz, C. M. Marian, and L. Karlsson, Chem. Phys. 229, 203 (1998).

[27] Z. Bao, R. Fink, O. Travnikova, D. Céolin, S. Svensson, and M. Piancastelli, J. Phys. B 41, 125101 (2008).

[28] T. Weber, M. Weckenbrock, M. Balser, L. Schmidt, O. Jagutzki, W. Arnold, O. Hohn, M. Schöffler, E. Arenholz, T. Young et al., Phys. Rev. Lett. 90, 153003 (2003).

[29] G. Prümper, H. Fukuzawa, D. Rolles, K. Sakai, K. C. Prince, J. R. Harries, Y. Tamenori, N. Berrah, and K. Ueda, Phys. Rev. Lett. 101, 233202 (2008).

[30] D. Rolles, G. Prümper, H. Fukuzawa, X.-J. Liu, Z. D. Pešić, R. F. Fink, A. N. Grum-Grzhimailo, I. Dumitriu, N. Berrah, and K. Ueda, Phys. Rev. Lett. 101, 263002 (2008).

[31] X.-J. Liu, Q. Miao, F. Gel'mukhanov, M. Patanen, O. Travnikova, C. Nicolas, H. Ågren, K. Ueda, and C. Miron, Nat. Photonics 9, 120 (2014).

[32] S. Song, B. Ding, W. Xu, C. Nicolas, M. Patanen, S. Nandi, J. Bozek, C. Miron, Z. Xiao, and X.-J. Liu, Phys. Rev. A 99, 022511 (2019).

[33] C. Miron and P. Morin, Nucl. Instrum. Methods Phys. Res., Sect. A 601, 66 (2009).

[34] C. Miron, M. Simon, N. Leclercq, and P. Morin, Rev. Sci. Instrum. 68, 3728 (1997).

[35] K. L. Guen, D. Céolin, R. Guillemin, C. Miron, N. Leclercq, M. Bougeard, M. Simon, P. Morin, A. Mocellin, F. Burmeister, A. N. de Brito, and S. L. Sorensen, Rev. Sci. Instrum. 73, 3885 (2002)

[36] X.-J. Liu, C. Nicolas, and C. Miron, Rev. Sci. Instrum. 84, 033105 (2013).

[37] G. Prümper and K. Ueda, Nucl. Instrum. Methods Phys. Res. Sect. A 574, 350 (2007).

[38] R. Dörner, T. Weber, M. Weckenbrock, A. Staudte, M. Hattass, H. Schmidt-Böcking, R. Moshammer, and J. Ullrich, Adv. At. Mol. Opt. Phy. 48, 1 (2002). 
[39] J. Ullrich, R. Moshammer, A. Dorn, R. Dörner, L. P. H. Schmidt, and H. Schmidt-Böcking, Rep. Prog. Phys. 66, 1463 (2003).

[40] Atomic Spectra Database, https://www.nist.gov/pml/atomicspectra-database (2016).
[41] E. Kukk, G. Snell, J. D. Bozek, W.-T. Cheng, and N. Berrah, Phys. Rev. A 63, 062702 (2001).

[42] E. Kukk, K. Ueda, U. Hergenhahn, X.-J. Liu, G. Prümper, H. Yoshida, Y. Tamenori, C. Makochekanwa, T. Tanaka, M. Kitajima, and H. Tanaka, Phys. Rev. Lett. 95, 133001 (2005). 